TECHNICAL NOTE

\author{
J. Sun \\ J. Wang \\ L. Jie \\ H. Wang \\ X. Gong
}

\section{Visualization of the Internal Cerebral Veins on MR Phase-Sensitive Imaging: Comparison with 3D Gadolinium-Enhanced MR Venography and Fast- Spoiled Gradient Recalled Imaging}

SUMMARY: The purpose of this article is to compare the visualization quality of ICV and their tributaries on PSI-MRV, 3D CE-MRV, and 3D CE-FSPGR. PSI-MRV has been shown to delineate the ICV, TSV, SV, anterior caudate nucleus veins, and medial atrial veins effectively. Due to its advantages of no contrast material injection, acceptable examination times, and no arterial contamination, PSIMRV should be considered in the evaluation of deep cerebral veins.

ABBREVIATIONS: 3D CE-FSPGR $=3 \mathrm{D}$ contrast-enhanced fast-spoiled gradient recalled imaging; 3D CE-MRV = 3D contrast-enhanced MR venography; ICV = internal cerebral veins; MinIP = minimum intensity projection; MIP = maximum intensity projection; $\mathrm{MRV}=\mathrm{MR}$ venography; $\mathrm{PSI}=$ phase-sensitive imaging; PSI-MRV = phase-sensitive imaging-based MR venography; $S V$ = septal veins; $S W I=$ susceptibility-weighted imaging; $T S V=$ thalamostriate veins
D eep cerebral veins have received increasing attention due to their importance in preoperational planning. ${ }^{1,2}$ Various MRV technologies have been developed for imaging the deep cerebral veins, including the ICV, noninvasively. However, displaying the ICV and their tributaries is still a challenge for noncontrast or contrast MRV, though the image quality has improved with the advent of higher field systems. ${ }^{3,4}$ SWI uses the paramagnetic deoxygenated hemoglobin in veins, which causes a shift in resonance frequency between the venous vessel and surrounding tissue, as an intrinsic contrast agent. In SWI-based venography, high spatial resolution increases the venous blood volume fraction within a voxel and leads to a better visualization of small venous structures. ${ }^{5,6} \mathrm{SWI}$ is called PSI, as implemented on the Signa Excite HD scanner (GE Healthcare, Milwaukee, Wisconsin). ${ }^{7}$ The purpose of this study was to evaluate the applicability of PSI-MRV in detecting the ICV and their tributaries through a comparison with 3D CE-MRV and 3D CE-FSPGR.

\section{Technique}

\section{Patients}

After obtaining the approval of the hospital ethics committee and written informed consent of patients, 65 consecutive patients with supratentorial meningioma were examined with 3D CE-MRV for presurgical evaluation of intracranial dural sinuses. PSI-MRV and 3D CE-FSPGR were performed before and after 3D CE-MRV.

Received May 23, 2010; accepted after revision August 14.

From the Department of Radiology (J.S., J.W., L.J.), The Second Affiliated Hospital, Zhejiang University College of Medicine, Hangzhou, China; Department of Radiology (X.G.), Sir Run Run Shaw Hospital, Zhejiang University College of Medicine, Hangzhou, China; and GE Healthcare (H.W.), Shanghai, China.

This study was supported by the Science and Technology Department of Zhejiang Province, China (No. 2009R10009).

Please address correspondence to Xiangyang Gong, MD, PhD, Department of Radiology, Sir Run Run Shaw Hospital, Zhejiang University College of Medicine, 3 Qingchun Dong Rd, Hangzhou, China, 310016; e-mail: cj.gxy@hotmail.com

Indicates open access to non-subscribers at www.ajnr.org

http://dx.doi.org/10.3174/ajnr.A2308
Twelve patients were excluded because the meningioma caused severe distortion of the third or lateral ventricular area. Nine patients were excluded because motion artifacts occurred in at least 1 of the 3 MRV examinations. Four patients who underwent repeat surgery were excluded because their images could not be evaluated due to susceptibility artifacts caused by hemorrhage around the ventricular area. A total of 40 patients (21 women and 19 men; age range, 22-77 years; mean age, 53.9 years) were enrolled.

\section{Imaging Examinations}

All MR imaging studies were performed on a 3T system (Signa Excite HD, GE Healthcare) equipped with an 8-channel phased array head coil. The MR imaging scanning field covered the bilateral hemispheres, including the ICV and their main tributaries. After conventional T1-weighted and T2-weighted imaging, PSI-MRV, 3D CE$\mathrm{MRV}$, and 3D CE-FSPGR were performed in order. 3D CE-MRV consisted of 2 scans with an acquisition time of 59 seconds each. A dose of $0.05-\mathrm{mmoL} / \mathrm{kg}$ gadolinium contrast material was administered intravenously with an injector at a rate of $3 \mathrm{~mL} / \mathrm{s}$ immediately after the first scan. A repeat scan with the same parameters was obtained after a 20-second delay. 3D CE-FSPGR was performed after the 3D CE-MRV sequence. The imaging parameters are listed in Table 1.

\section{Image Reconstruction}

The MR images were postprocessed with an Advantage Workstation version 4.2 (GE Healthcare). For PSI-MRV, images were reformatted

Table 1: Imaging parameters in PSI, 3D CE-MRV, and 3D CE-FSPGR sequences

\begin{tabular}{lccc}
\hline Parameter & PSI & 3D CE-MRV & 3D CE-FSPGR \\
\hline Orientation & Axial & Sagittal & Axial \\
TR (ms) & 40 & 3.1 & 7.8 \\
TE $(\mathrm{ms})$ & 26 & 0.9 & 3.8 \\
Flip angle & $20^{\circ}$ & $25^{\circ}$ & $25^{\circ}$ \\
Slab thickness $(\mathrm{mm})$ & 80 & 160 & $160-180$ \\
Section thickness $(\mathrm{mm})$ & 2 & 1 & 0.7 \\
FOV $(\mathrm{mm})$ & 240 & 260 & 240 \\
Pixel matrix & $320 \times 288$ & $288 \times 256$ & $320 \times 256$ \\
NEX & 0.75 & 1 & 2 \\
Acquisition time $(\mathrm{min} / \mathrm{sec})$ & 4.57 & 1.50 & $5.47-7.43$ \\
\hline
\end{tabular}


Table 2: A comparison of the visualization of ICV and tributaries on the 3 different MRV techniques

\begin{tabular}{|c|c|c|c|c|c|c|c|c|c|}
\hline \multirow[b]{3}{*}{ Veins } & \multicolumn{6}{|c|}{ MRV } & \multirow{3}{*}{$\begin{array}{l}\text { PSI-MRV/ } \\
\text { 3D CE-MRV }\end{array}$} & \multirow{3}{*}{$\begin{array}{c}\text { PSI-MRV/ } \\
\text { 3D CE-FSPGR }\end{array}$} & \multirow{3}{*}{$\begin{array}{l}\text { 3D CE-MRV/ } \\
\text { 3D CE-FSPGR }\end{array}$} \\
\hline & \multicolumn{2}{|c|}{ PSI-MRV } & \multicolumn{2}{|c|}{ 3D CE-MRV } & \multicolumn{2}{|c|}{ 3D CE-FSPGR } & & & \\
\hline & Range & Mean & Range & Mean & Range & Mean & & & \\
\hline ICV & $3-3$ & $3.00 \pm 0.00$ & $3-3$ & $3.00 \pm 0.00$ & $3-3$ & $3.00 \pm 0.00$ & None & None & None \\
\hline TSV & $3-3$ & $3.00 \pm 0.00$ & $3-3$ & $3.00 \pm 0.00$ & $3-3$ & $3.00 \pm 0.00$ & None & None & None \\
\hline SV & $2-3$ & $2.90 \pm 0.30$ & $1-3$ & $2.44 \pm 0.52$ & $2-3$ & $2.73 \pm 0.50$ & $\mathrm{PSI}^{\mathrm{a}}$ & $\mathrm{PSI}^{\mathrm{a}}$ & FSPGR $^{a}$ \\
\hline $\begin{array}{l}\text { Anterior caudate nucleus } \\
\text { veins }\end{array}$ & $1-3$ & $2.34 \pm 0.81$ & $0-3$ & $1.44 \pm 0.74$ & $1-3$ & $2.46 \pm 0.57$ & $\mathrm{PSI}^{\mathrm{a}}$ & FSPGR $^{\mathrm{b}}$ & FSPGR $^{\mathrm{a}}$ \\
\hline Superior choroidal vein & $0-1$ & $0.86 \pm 0.35$ & $0-2$ & $1.65 \pm 0.66$ & $0-2$ & $1.85 \pm 0.51$ & 3D CE-MRV ${ }^{a}$ & FSPGR $^{a}$ & FSPGR $^{a}$ \\
\hline Medial atrial vein & $0-3$ & $1.93 \pm 0.94$ & $0-3$ & $1.50 \pm 0.91$ & $0-3$ & $2.09 \pm 1.04$ & $\mathrm{PSI}^{\mathrm{a}}$ & FSPGR $^{\mathrm{c}}$ & FSPGR $^{\mathrm{c}}$ \\
\hline
\end{tabular}

a $P<.001$.

b $P<.01$

c $P<.05$.

by using the MinIP technique with 15 - to 30 -mm thickness in the transverse plane. Reconstructed images were saved once the ICV and tributaries were optimally displayed. A subtraction was performed between the pre- and postcontrast 3D CE-MRV scannings to get images without background pollution. The subtracted images were subsequently reconstructed in the transverse plane by using an MIP algorithm. The MIP images used the same thickness and orientation as the prior PSI-MRV images. 3D CE-FSPGR images were also reformatted with the MIP algorithm in the transverse plane like the 3D CE-MRV images. The same technician performed all image reconstructions.

\section{Image Evaluation}

The reconstructed images of PSI-MRV, 3D CE-MRV, and 3D CEFSPGR were evaluated by a consensus of 2 radiologists with previous experience in PSI and CE-MRV. Source images were reviewed when the radiologists needed to trace or define a vein. Evaluation from another experienced radiologist was obtained when the 2 reviewers failed to agree. The veins evaluated in this study included the ICV, TSV, SV, anterior caudate nucleus vein, superior choroidal vein, and medial atrial vein. Visualization of the veins was qualitatively graded in 4 levels: intense and continuous $=3$, faint and continuous $=2$, partially visible $=1$, invisible $=0$. The Wilcoxon signed rank test was used to analyze differences in the visualization quality of the ICV and its tributaries among the 3 MRV techniques.

\section{Results}

The ICV and TSV were clearly and completely displayed at low signal intensity with PSI-MRV in all 80 lateral hemispheres of the 40 patients. They were equally well shown on the 3D CEMRV and 3D CE-FSPGR images. The SV were delineated best on PSI-MRV, with an average score of 2.9 , followed by $3 \mathrm{D}$ CE-FSPGR (2.73) and then 3D CE-MRV (2.44) (Table 2 and Fig $1 A-C)$. There were significant differences among the 3 MRV modalities ( $P<.001$ for each pair of MRV images). Anterior caudate nucleus veins were shown qualitatively better on PSI-MRV (2.34) than on 3D CE-MRV (1.44) with significant differences $(P<.001)$, but inferior to the veins on $3 \mathrm{D}$ CE-FSPGR (2.46) $(P<.01)$. The superior choroidal vein was poorly delineated in all patients on PSI-MRV, with an average score of 0.86 , which was inferior to scores of veins demonstrated on the 3D CE-MRV or 3D CE-FSPGR $(P<.001$ for each pair) (Table 2 and Fig $2 A-C$ ). For visualization of the medial atrial vein, PSI-MRV was better than 3D CE-MRV
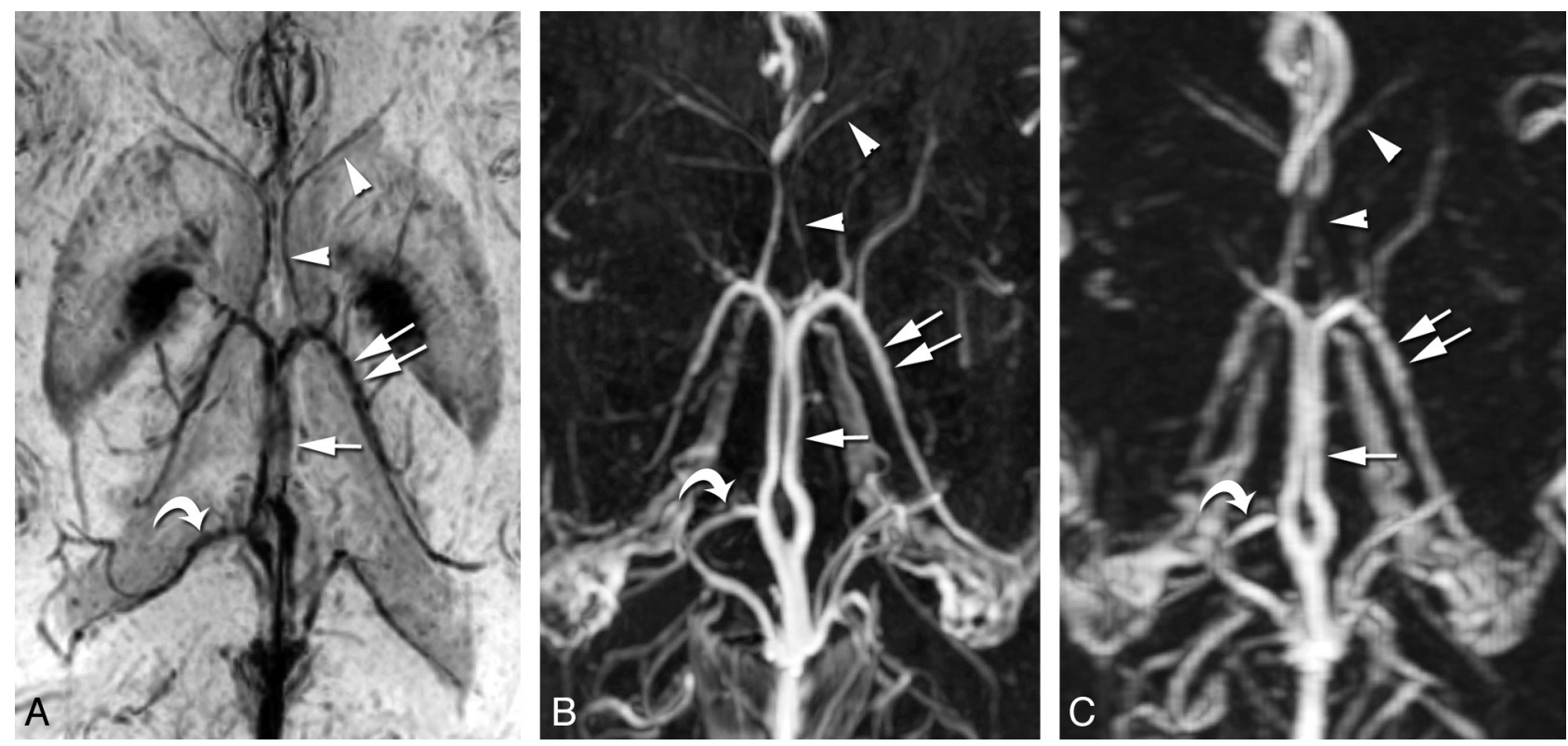

Fig 1. The ICV (arrow) and TSV (double arrows) are clearly and completely displayed with the PSI-MRV (A), 3D CE-FSPGR (B), and 3D CE-MRV (C) in this patient. The septal veins (arrowheads) are delineated best on the PSI-MRV, followed by the 3D CE-FSPGR and then the 3D CE-MRV. The medial atrial vein (curved arrows) is easily identified on PSI-MRV, compared with 3D CE-FSPGR and 3D CE-MRV, because of the easy localization of the lateral ventricles and less arterial pollution around the vein of Galen. 

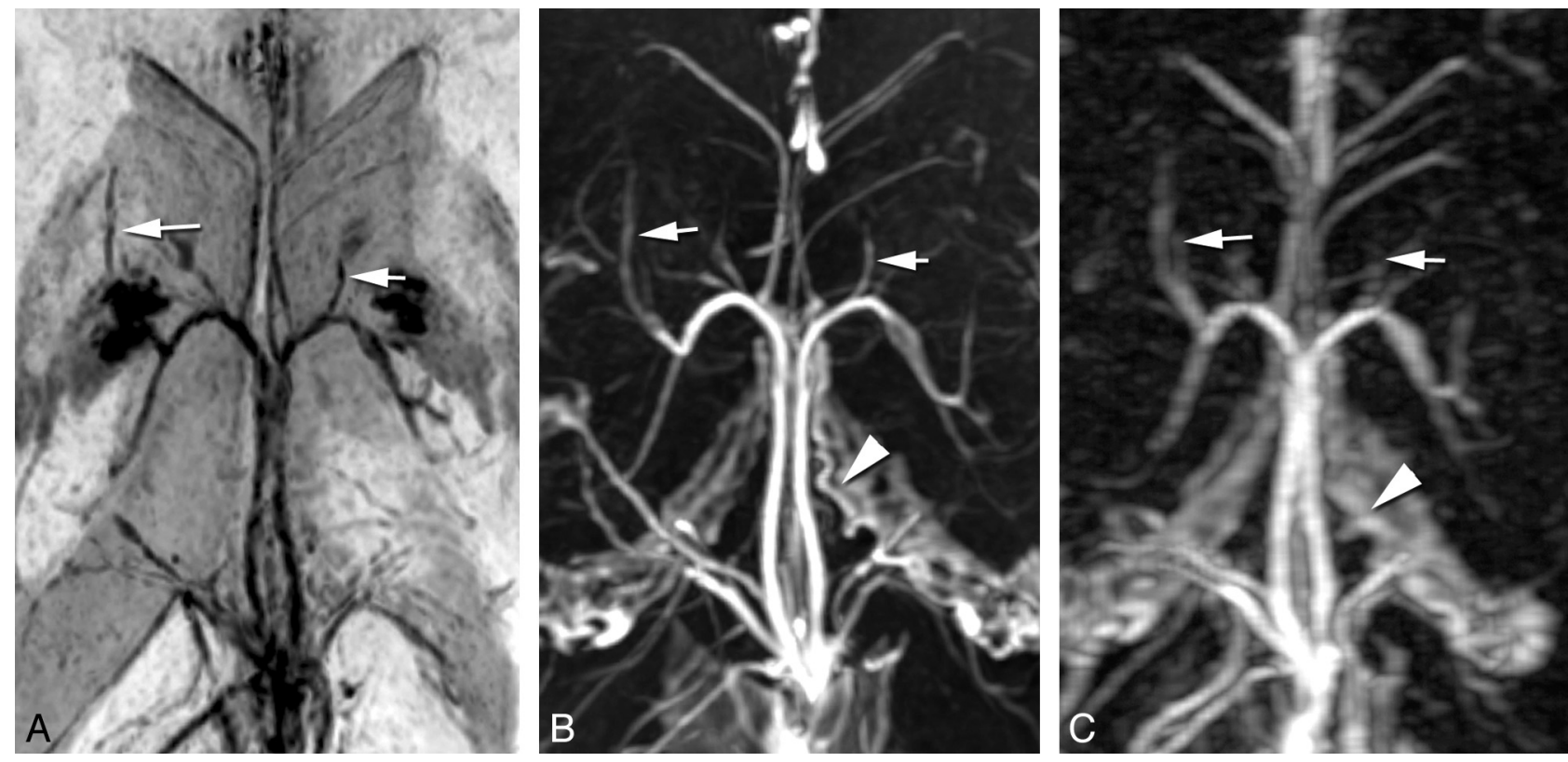

Fig 2. Anterior caudate nucleus veins (arrow) are shown qualitatively best on 3D CE-FSPGR (B), followed by PSI-MRV (A), and then 3D CE-MRV (C). The superior choroidal vein (arrowhead) is clearly shown on 3D CE-FSPGR $(B)$, poorly shown on 3D CE-MRV $(C)$, and invisible on PSI-MRV $(A)$ in this patient.

$(P<.001)$ but worse than 3D CE-FSPGR, with a statistical difference $(P<.05)$.

\section{Discussion}

On the basis of similar principles of SWI, PSI has been introduced to evaluate the variants of ICV noninvasively. ${ }^{8}$ With this study, all 3 MRV techniques provided equally good demonstration of the ICV and TSV. The SV were best shown with PSI-MRV among the $3 \mathrm{MRV}$ procedures. The anterior caudate nucleus veins, which comprised several small veins draining inward and backward from the head of the caudate nucleus to the TSV, SV, or ICV, were observed on PSI-MRV with a better image quality than on 3D CE-MRV. The medial atrial vein runs along the medial wall of the atrium and joins the ICV at its terminal portion. With 3D CE-MRV and 3D CE-FSPGR, this vein was commonly confused with other vessels around the vein of Galen. Thus, the reviewers had to carefully identify the vein with the aid of the original image. However, it was quite easy to identify this vein on PSI-MRV because PSI-MRV showed the lateral ventricles clearly and reduced pollution from small arteries around the vein of Galen. The PSI-MRV display of the superior choroidal vein was poor compared with the 3D CE-MRV and 3D CE-FSPGR sequences, possibly because CSF signals obscure the vessel passing the ventricle in PSI MinIP images.

Compared with 3D CE-MRV and 3D CE-FSPGR, using PSI to evaluate deep cerebral veins has several advantages. First, no injection of contrast material is required on PSIMRV. Second, the PSI-MRV image is free from arterial contamination, which is a big issue on 3D CE-MRV and 3D CEFSPGR. Finally, the examination can be repeated immediately if the MRV fails for various reasons, because PSI scanning is free of any contamination from brain parenchymal or meningeal contrast enhancement. The examination duration for PSI-MRV is relatively longer than for 3D CE-MRV, but it is almost commensurate with 3D CE-FSPGR. Most patients can tolerate the examination duration.
The basal vein of Rosenthal, another important component of the deep cerebral veins, was not included in this study. A 2-mmthickness image paralleling the ICV was insufficient to show this vein because it forms an angle to the ICV. An additional acquisition is needed for evaluating the basal vein of Rosenthal. The original image of 3D CE-MRV in this study was designed in the sagittal plane, which enables reliable visualization of the relationship between a meningioma and the superior sagittal sinus. The in-plane spatial resolution of the reconstructed transverse images may underestimate the small tributaries of ICV.

\section{Acknowledgment}

We thank Mrs. Trudie Loo for her written polishing of this article.

\section{References}

1. Giordano M, Wrede KH, Stieglitz LH, et al. Identification of venous variants in the pineal region with $3 \mathrm{D}$ preoperative computed tomography and magnetic resonance imaging navigation: a statistical study of venous anatomy in living patients. J Neurosurg 2007;106:1006-11

2. Kiliç T, Ozduman K, Cavdar S, et al. The galenic venous system: surgical anatomy and its angiographic and magnetic resonance venographic correlations. Eur J Radiol 2005;56:212-19

3. Kirchhof K, Welzel T, Jansen Q, et al. More reliable noninvasive visualization of the cerebral veins and dural sinuses: comparison of three MR angiographic techniques. Radiology 2002;224:804-10

4. Lettau M, Sartor K, Heiland S, et al. 3T high-spatial-resolution contrast-enhanced MR angiography of the intracranial venous system with parallel imaging. AJNR Am J Neuroradiol 2009;30:185-87

5. Reichenbach JR, Venkatesan R, Schillinger DJ, et al. Small vessels in the human brain: MR venography with deoxyhemoglobin as an intrinsic contrast agent. Radiology 1997;204:272-77

6. Reichenbach JR, Jonetz-Mentzel L, Fitzek C, et al. High-resolution blood oxygen-level dependent MR venography (HRBV): a new technique. Neuroradiology 2001;43:364-69

7. Kurosaki M, Tabuchi S, Akatsuka K, et al. Application of phase sensitive imaging (PSI) for hemorrhage diagnosis in pituitary adenomas. Neurol Res 2010;32:614-19

8. Fujii S, Kanasaki Y, Matsusue E, et al. Demonstration of cerebral venous variations in the region of the third ventricle on phase-sensitive imaging. AJNR Am J Neuroradiol 2010;31:55-59 